\title{
Different timing in allograft and autograft maturation after primary anterior cruciate ligament reconstruction does not influence the clinical outcome at mid-long-term follow-up
}

\author{
Andrea Cusumano ${ }^{1}$. Paolo Capitani ${ }^{2}$. Carmelo Messina ${ }^{1}$. Laura de Girolamo ${ }^{1}$ - Marco Viganò ${ }^{1}$. Giovanni Ravasio ${ }^{1}$. \\ Francesca Facchini ${ }^{1} \cdot$ Luca Maria Sconfienza $^{1,3} \cdot$ Alberto Zerbi $^{4} \cdot$ Herbert Schoenhuber $^{1} \cdot$ Roberto Pozzoni $^{1}$. \\ Gabriele Thiébat ${ }^{1}{ }^{1}$
}

Received: 6 April 2021 / Accepted: 25 October 2021 / Published online: 16 November 2021

(c) The Author(s) under exclusive licence to European Society of Sports Traumatology, Knee Surgery, Arthroscopy (ESSKA) 2021

\begin{abstract}
Purpose The use of allografts in primary anterior cruciate ligament reconstruction (ACLR) is increasing although they are still supposed to be associated to greater risk of re-rupture due to a slower and less efficient graft maturation. The aim of this prospective randomized controlled study was to compare the graft maturation after ACLR with allograft and autograft by MRI at 6- and 12-month follow-up and integrate these data with the functional and clinical results observed at 6-, 12- and 60-month follow-up.

Methods Fifty patients with indication to primary ACLR were randomly and equally divided into hamstring autograft or allograft tendon groups. The graft maturation was measured at 6- and 12-month follow-up by the SNQ score and other radiological parameters on MRI scans. Clinical and functional recovery was evaluated by Lysholm score, Visual Analogues Scale, Tegner activity scale and modified Cincinnati knee rating system at 6,12 and 60 months after surgery to estimate the predictive value of the radiological parameters for clinical outcomes. Return-to-sport (ACL-RSI) was measured 60 months after surgery.

Results Three patients had retear of the neo-ligament (two from Auto group and one from Allo group). All the clinical/ functional parameters significantly improved over time, with no statistically significant difference between the groups. At 6 months, the SNQ value was significantly higher in the Auto than in the Allo group (12.9 vs 7.9, $p=0.038)$, but at 12 months they were comparable (9.8 vs 10.4). The 6-month SNQ values did not correlate with the clinical scores, whereas the 12-month SNQ values significantly correlated with the Cincinnati score, Lysholm score and Tegner activity scale collected at 60-month follow-up.

Conclusion No clinical or functional differences have been found between the two treatment groups, supporting the suitability of using allograft in primary ACLR, when available. The results at MRI scans showed a different graft maturation trend in the two groups, with allografts being more reactive in the first 6 months. MRI together with the subjective evaluation allows to evaluate objectively the status of the neo-ligamentization process and therefore helps the surgeon to dictate the individual time for return-to-sport.
\end{abstract}

Level of evidence Level I.

Keywords ACL $\cdot$ Anterior cruciate ligament $\cdot$ Reconstruction $\cdot$ Autograft $\cdot$ Allograft $\cdot$ SNQ $\cdot$ MRI $\cdot$ Graft Maturation

\section{Introduction}

Gabriele Thiébat

gthiebat@gmail.com

Extended author information available on the last page of the article
Anterior cruciate ligament reconstruction (ACLR) with the patient's own autograft tissue (hamstring or bone patellar tendon bone) is the most common surgical technique to treat ACL complete rupture. Allografts are commonly used in revision and multiligament surgery [30]; albeit, in the last 
decades, their use also in primary ACLR is growing $[1,13$, 36].

Despite the advantages of allografts in terms of reduced surgical time and donor site morbidity, they are not commonly accepted for primary ACLR, being thought to be associated with increased infectious risk and poorer clinical outcomes.

However, more recent systematic reviews have shown that non-irradiated and non-chemically treated allografts produce comparable results to autografts in terms of patient satisfaction, knee functional score and clinical failure rate [13, 15, 22, 36-38], although they are still thought to have a greater risk of re-rupture, particularly in young and active patients [9].

Beyond these data, given the paucity in some countries of allogenic material and the related costs, the use of allograft in primary ACLR is under discussion, especially analyzing the possible advantages to return to sport [13]. More recently, the European Society of Sports Traumatology Knee Surgery and Arthroscopy (ESSKA) has dedicated a whole initiative to evaluate the availability, awareness and cost-effectiveness of allograft tissue in joint reconstruction in Europe [13, 29].

While knee functional score and clinical failure rate after allograft ACLR have been investigated and compared, an accurate and complete radiological evaluation of the possible differences between autograft and allograft in term of maturation and integration of neo-ligament is still lacking $[8,11]$. These objective parameters are known to be strongly correlated to the long-term stability of the knee and, therefore, could give additional information to direct the surgeons' choice [19]. Although not commonly adopted during postoperative follow-up, magnetic resonance imaging (MRI) is the preferred and most commonly used technique to morphologically evaluate graft remodeling [10].

The primary aim of this prospective, single-center randomized single-blinded study was to evaluate radiologically the maturity of the neo-ACL tendon graft after 6 and 12 months from surgery. The secondary aim was to evaluate the clinical-functional recovery of daily activities (work and recreation), integrating these data with the radiological results, to provide recommendation on the use of allograft in primary ACLR. Moreover, the patients were clinically re-evaluated at 5-year follow-up, in order to assess any clinical differences between patients treated by either autograft or allograft ACL reconstruction and to estimate the predictive value of radiological parameters for mid-term clinical outcomes. The thorough radiological evaluation in the first phases of graft maturation, accompanied by simultaneous and long-term clinical evaluations, bring additional information to the existing one about the suitability of using allograft in primary ACLR as a reasonable alternative to hamstring autografts.

\section{Materials and methods}

This single-center, interventional, prospective, randomized, controlled, single-blinded study was approved by the Ethics Committee (27/INT/2014) and performed according to the standards EN ISO 14155:1, EN ISO 14155:2, Declaration of Helsinki and Good Clinical Practices (GCP).

\section{Patient enrollment}

Patients with a primary and unilateral ACL complete tear with indication to ACLR were considered eligible for the study. Fifty patients who met the inclusion criteria were asked to sign the informed consent and were randomly assigned into one of the two study groups: Autograft Group (Auto, 25 patients) and Allograft Group (Allo, 25 patients). Inclusion criteria were radiological and clinical diagnosis of complete primary and unilateral ACL tear, male or female, age between 18 and 40 years old, Tegner activity level pre-injury $>5$, time from injury between 90 and 270 days. Any previous knee surgery, chondral lesion ICRS grade 2 or higher, complete meniscal injury, osteoarthritis even at initial phase, immune-mediated diseases, systemic chronic inflammatory disorders or knee/ankle infections, cardiac, pulmonary, neurological or other diseases that may increase the operative risk, use of drugs associated to increased risk of tendinitis and tendon ruptures, pregnancy status or breast feeding were considered as exclusion criteria.

The mean age was $27 \pm 7$ y/o in Auto group and $30 \pm 6$ y/o in Allo group. The first one was composed by 21 men $(88 \%)$ and 3 women (12\%), whereas the second by 23 men (92\%) and 2 women (8\%). There were $42 \%$ left and $58 \%$ right knees, and $36 \%$ left and $64 \%$ right in group Auto and Allo, respectively. No statistically significant differences in term of population's characteristics were observed between the two groups.

\section{Surgical procedure}

All the procedures were performed by two experienced surgeons with the same single-bundle transtibial arthroscopic technique, preserving the ACL remnant tissue. When present, associated injuries were treated first to confirm full adherence to the inclusion/exclusion criteria. 
Semitendinosus and gracilis tendons were used for all the patients of the Auto group.

For the patients of the Allo group, 17/25 (68\%) of the allograft used were peroneal tendons, 8/25 (32\%) posterior tibialis tendons. All the implanted allografts were nonirradiated fresh-frozen tissues, obtained from a single certified tissue bank.

Both autografts and allografts, doubled through the loop of the suspension system, were fixed to femoral surface by TightRope ${ }^{\circledR}$ metal plate and to the tibial bone by a bioabsorbable interference screw (Genesys ${ }^{\mathrm{TM}}$ Matryx ${ }^{\circledR}$, ConMed Corp) [3].

\section{Rehabilitation program}

No knee braces were prescribed during the postoperative period.

The patients of both groups followed the same rehabilitation protocol, with an initial partial load and progressive recovery of the range of motion accompanied by isometric muscle strengthening. Water exercises, exercise bike and proprioceptive gymnastics were then introduced, while muscle strengthening continued with closed kinetic chain exercises followed by open kinetic chain exercises. Unidirectional running was included during the third month; running with changes of direction, leaps and recovery of the sporting gesture during the fifth month. Return to sport was accorded at the end of the eighth/ninth month, after recovery of quadricipital trophism and excellent proprioceptive control [21].

\section{Clinical evaluation}

All the patients were clinically assessed by Lysholm score [5], Tegner Activity Scale [4], modified Cincinnati Knee Rating System [28] and Visual Analog Scale (VAS) [33] at the time of enrollment (T0) and then at 6 months (T1), at 12 months (T2) after surgery. At about 5 years from surgery (T3) Lysholm score, Tegner Activity Scale, modified Cincinnati knee rating system and ACL-RSI [32] were collected.

\section{MRI evaluation}

All the patients underwent MRI at T0, T1 and T2. The MRI scans were performed with a 1.5 Tesla scanner (MAGNETOM ${ }^{\circledR}$ Avanto, Siemens Medical Solution, Erlangen, Germany) following a standardized image acquisition protocol (Table 1). Each knee was scanned in slightly flexed position (about $10^{\circ}$ ) with a dedicated extremity coil.

Image analysis was focused on several parameters: The signal intensity (SI) of ACL graft was evaluated by calculating the signal-to-noise quotient (SNQ) [31]. The SI of the graft was measured at three different regions of interest (upper, middle and lower third), and the mean was normalized by measuring the SI of the distal quadriceps tendon (QT) (Fig. 1).

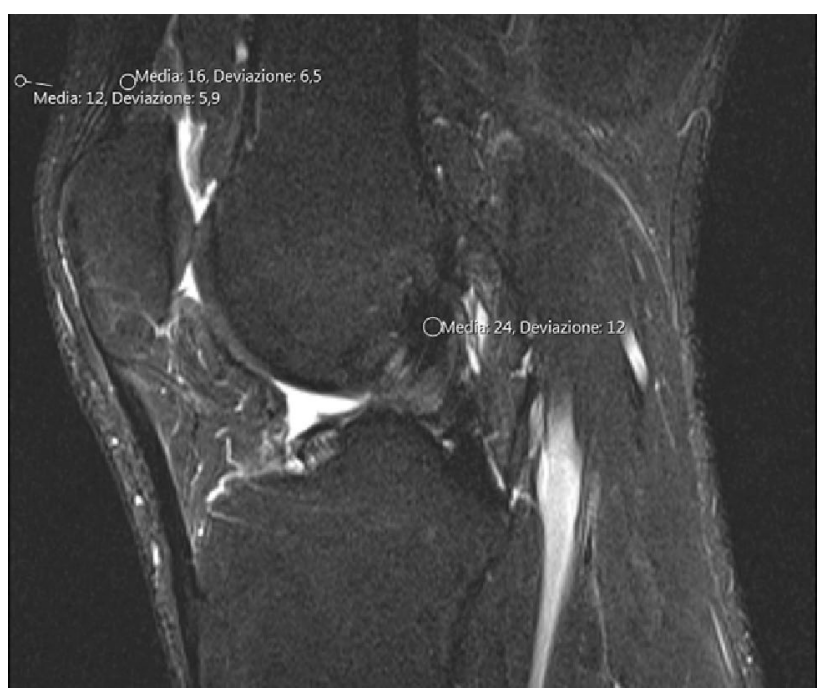

Fig. 1 MRI image showing the measurements used for SNQ calculation. The signal intensity (SI) of ACL graft was calculated using the signal-to-noise quotient (SNQ). The SI of the graft was measured at three different regions of interest (upper, middle and lower third), and the mean was normalized by measuring the SI of the distal quadriceps tendon (QT) "Media"= Mean, "Deviazione" = Standard Deviation
Table 1 MRI study protocol. An oblique STIR (para-sagittal) additionally to the conventional MRI protocol was included to better evaluate graft signal and its degree of edema

\begin{tabular}{|c|c|c|c|c|}
\hline & $\mathrm{TR} / \mathrm{TE}$ & Slice thickness & FOV & Averages \\
\hline Sagittal T1-weighted TSE & $500 / 13$ & $3 \mathrm{~mm}$ & $180 \mathrm{~mm}$ & 1 \\
\hline Sagittal FS proton density & $2840 / 29$ & $3 \mathrm{~mm}$ & $180 \mathrm{~mm}$ & 1 \\
\hline Oblique sagittal STIR & $\begin{array}{l}5420 / 30 \\
\quad(\mathrm{IT}=140 \mathrm{~ms})\end{array}$ & $3 \mathrm{~mm}$ & $170 \mathrm{~mm}$ & 1 \\
\hline Transverse T2 weighted TSE & $4100 / 104$ & $3 \mathrm{~mm}$ & $180 \mathrm{~mm}$ & 1 \\
\hline Transverse FS proton density & $3450 / 35$ & $3 \mathrm{~mm}$ & $180 \mathrm{~mm}$ & 2 \\
\hline Coronal T2 weighted TSE & $5450 / 108$ & $3 \mathrm{~mm}$ & $200 \mathrm{~mm}$ & 1 \\
\hline
\end{tabular}

$T R$ repetition time; TE echo time; FOV field of view; TSE turbo spin-echo; FS fat saturated; STIR short tau inversion recovery; $I T$ inversion time 
The background signal was measured to finally calculate the SNQ as follows:

$S N Q=\frac{\text { Graft } S I-Q T S I}{\text { Background } S I}$

The SNQ is capable of normalizing the SI of each graft in this study; therefore, in this study, the SNQ value was used to quantify the signal intensity emitted by the neo-ligament. The SNQ evaluates the tendon graft maturation, with low values indicating a greater maturation of the graft. Position of the tibial tunnel, orientation of the neo-ACL estimated using the sagittal angle, angle between the ACL and the Blumensaat line, edematous condition of the graft based on the short tau inversion recovery (STIR) sequence, using three different grades (grade I: normal signal, grade II: possible edema, grade III: $100 \%$ of edema), and mean width of the graft, were also measured in all sequences at three different sites (distal, intermediate, proximal) (Fig. 2).

MRI was evaluated by a senior radiologist who, as well as the clinician in charge of patients' evaluation, was blinded to the patients' group allocation.

\section{Statistical analysis}

Analysis was performed using R software version 4.0.3 (R Core Team, Wien, Austria).

Quantitative variables were described using the mean and standard deviation, whereas qualitative variables were reported with absolute and percentage frequencies.

Differences in categorical variables between groups were assessed by Chi-square test. For continuous variables, data distribution was assessed by Shapiro-Wilk test. In case of normally distributed data, one-way ANOVA (with Bonferroni's post-test) or Student's t test were applied, conversely Mann-Whitney and Kruskall-Wallis test (with Dunn's post hoc test) were used. Two-way ANOVA with Bonferroni's post-test was applied to assess the influence of two variables on the same parameter. Correlation tests were performed according to Pearson's or Spearman's method, depending on data distribution. Linear regression models were used to test the influence of multiple variables on continuous data, while logistic regression models were applied to categorical outcomes.

The sample size of the study was calculated from the SNQ index. Assuming a standard deviation (SD) of the SNQ value of 3 , a relevant difference between the two treatment groups in favor of the autograft group of 3 [8], a test power (1- $\beta$ ) of $90 \%$ and a two-tailed alpha error of $5 \%$, a sample size of 22 subjects per group was obtained.

Considering a possible loss of subjects in the follow-up of around $10 \%, 25$ patients were enrolled in each treatment group.

A value of $\mathrm{p}<0.05$ was considered statistically significant [7].

\section{Results}

None of the patients had adverse events neither in the perioperative nor in the immediate postoperative period.

\section{Clinical results}

In both groups, the most common associated lesion with ACL injury was lateral meniscus tear: five patients in the Auto group (all treated by partial lateral meniscectomy) and nine patients in Allo group (eight treated with partial lateral meniscectomy, one treated with out-in suture repair). Medial meniscus tear was found in two patients of Auto group (one treated with partial medial meniscectomy, one treated with out-in suture repair) and five patients of Allo group (one treated with partial medial meniscectomy, four treated with out-in suture repair).

One patient of the Auto group had a neo-ACL re-rupture because he resumed the full sport activity too early (4 months after surgery) without medical authorization and therefore he was excluded from the study. Likewise, two more patients were excluded from the study because of the neo-ACL re-rupture, one of the Auto group and one of the Allo group, 37 and 30 months from surgery, respectively. Moreover, two patients of the Allo group were excluded
Fig. 2 Methods used to measure the mean width of the graft on sagittal MRI at femoral, intraarticular and tibial level
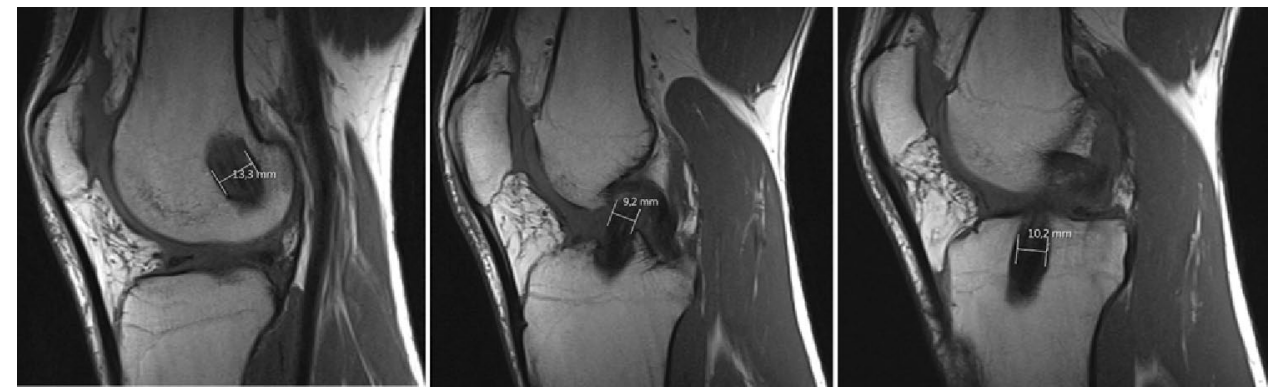
during the first year after surgery for reasons unrelated to the study.

Therefore, 47 patients out of the 50 initially enrolled were available at 6 and 12 months follow-up and 45 for the last follow-up analysis ( $63 \pm 7$ months).

Other minor complications occurred in both groups but they did not lead to the exclusion of these patients from the study (Supplementary Table 1).

All the clinical and functional scores used for patient evaluation showed no significant differences between the two groups at any time points (Supplementary Table 2).

At the last follow-up, 44 out of 45 patients were satisfied with the surgical outcome and they declared they would undergo the same treatment. Fourty-one patients ( 22 of the Auto group and 19 of the Allo group) reported knee stability in every situation, whereas 4 patients (one of the Auto group and three of the Allo group) had occasional instability during intense activities, such as demanding sports and/or heavy weightlifting. No patient complained about knee instability during daily activities.

The Lysholm score improved over time, with no differences between the study groups (n.s.; interaction n.s.) (Fig. 3A). The same behaviour was observed for the Cincinnati score (Fig. 3B) and Tegner activity scale (Fig. 3C). Tegner Actvity Scale showed particularly relevant reductions at 6-month follow-up, due to the activity restrictions in both groups during the rehabilitation period.

VAS showed a decrease during the first year in both groups, reaching a significant difference at 12 months

\section{ACL-RSI}

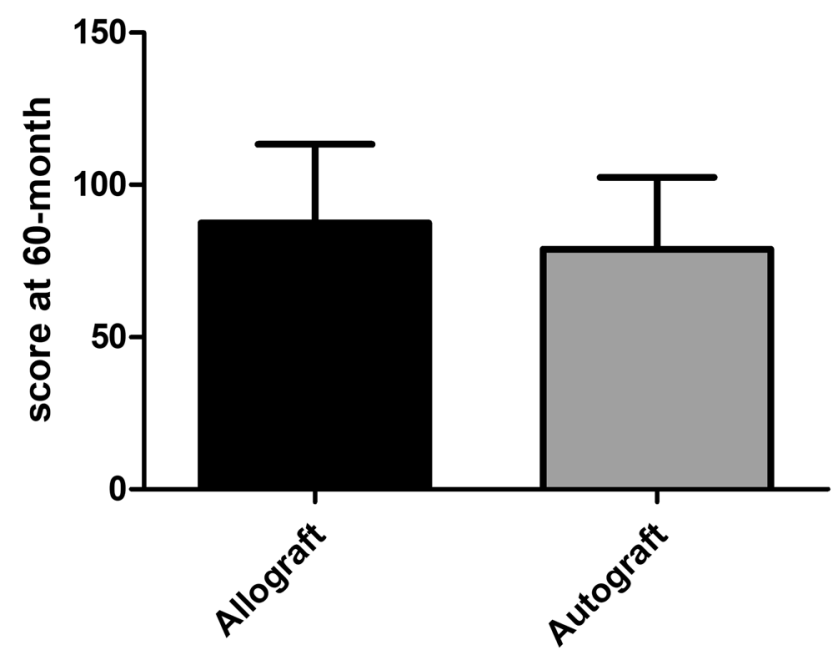

Fig. 4 ACL-RSI score at the 60-month follow-up in the Allo and Auto groups, showing no difference between the two groups

$(p=0.014)$, with new non-significant increases at 60-month follow-up with respect to 12-month (Fig. 3D).

The ACL-RSI collected 60 months after surgery did not differ between patients in the autograft and allograft groups (n.s.) (Fig. 4).
Fig. 3 Functional, pain and activity scores at the different time points in Allograft and Autograft groups. Lysholm Score (A); Cincinnati Score (B); Tegner Activity Scale (C); Visual Analogue Score-Pain (D). With the exception of VAS that only improved at 12 -month follow-up, significant differences were observed for all the other parameters between either baseline levels and/or 6-month follow-up with respect to each time point. No difference was observed between the two groups. $* p<0.05, * * * p<0.001$ vs baseline; ${ }^{\#} p<0.05$, ${ }^{\# \#} p<0.01,{ }^{\# \# \#} p<0.001$ vs 6 months a

LYSHOLM

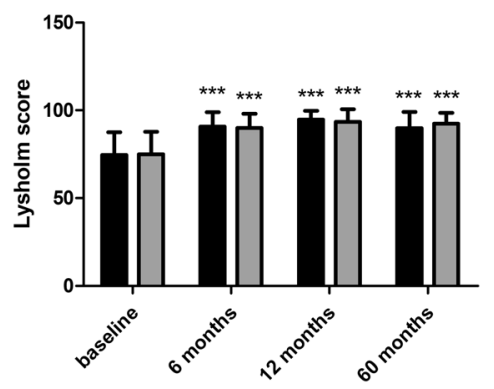

C

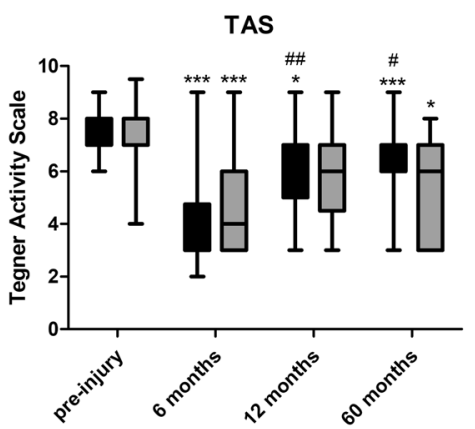

b

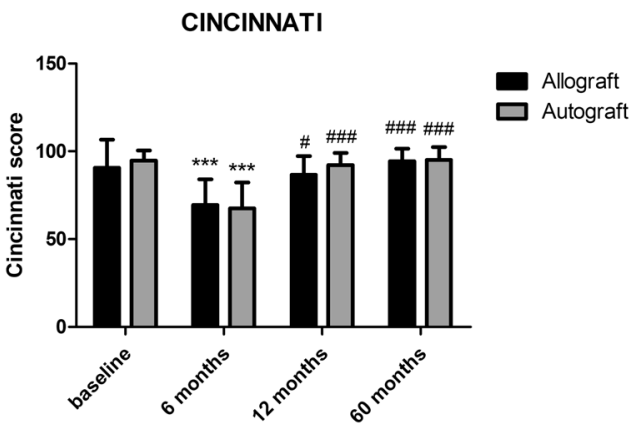

d

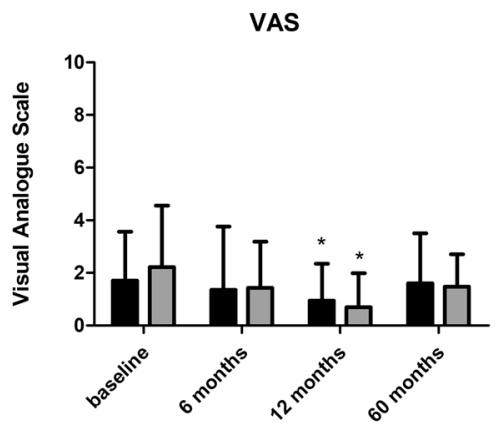




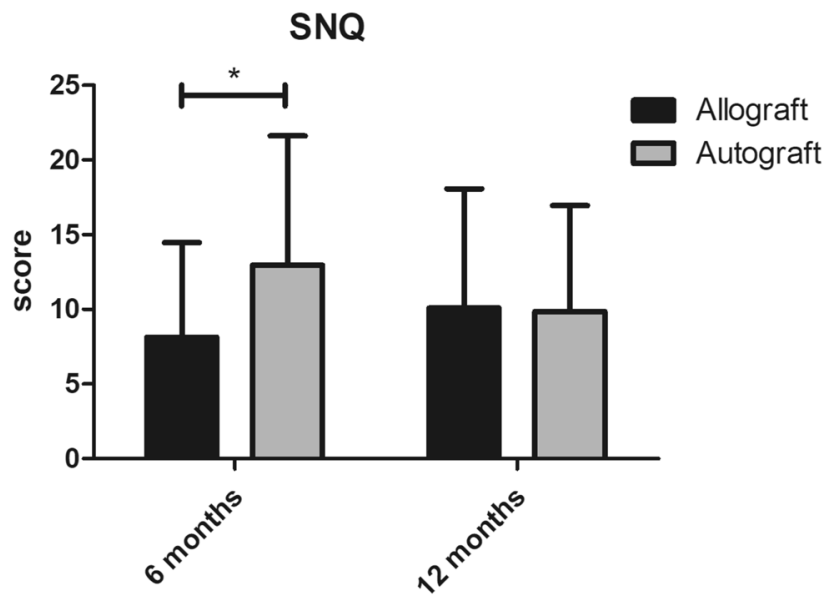

Fig. 5 Comparison of the SNQ score between Allo and Auto groups. Data are presented as mean $\pm \mathrm{SD} ;{ }^{*} p<0.05$. At 6 months the mean SNQ index was significantly higher in the Auto group than in the Allo group $(p=0.038)$, whereas at 12 months the values were comparable. The two-way ANOVA analysis indicated a different behavior during time depending on the graft type

Table 2 Full list of MRI measurements at femoral and tibial site, with corresponding p-values for autograft and allograft groups

\begin{tabular}{|c|c|c|c|c|c|}
\hline & \multicolumn{2}{|c|}{$\begin{array}{l}\text { Autograft } \\
\text { group }\end{array}$} & \multicolumn{2}{|c|}{$\begin{array}{l}\text { Allograft } \\
\text { group }\end{array}$} & \multirow[t]{2}{*}{$p$ value } \\
\hline & Mean & $S D$ & Mean & $S D$ & \\
\hline $\begin{array}{l}\text { Posterior-anterior position ratio } \\
6 \mathrm{~m}\end{array}$ & 56.7 & 7.2 & 54.8 & 8.4 & ns \\
\hline $\begin{array}{l}\text { Posterior-anterior position ratio } \\
12 \mathrm{~m}\end{array}$ & 56.6 & 7.3 & 55.9 & 8.2 & ns \\
\hline Medial-lateral position ratio $6 \mathrm{~m}$ & 41.3 & 3.2 & 39.0 & 2.7 & 0.01 \\
\hline Medial-lateral postition ratio $12 \mathrm{~m}$ & 41.8 & 3.3 & 38.7 & 3.6 & 0.003 \\
\hline Femoral 6 m width (mm) & 9.2 & 1.0 & 8.6 & 0.8 & 0.012 \\
\hline Femoral 12 m width (mm) & 9.4 & 1.1 & 8.7 & 1.0 & 0.019 \\
\hline Articular 6 m width $(\mathrm{mm})$ & 8.7 & 0.6 & 8.3 & 0.6 & 0.025 \\
\hline Articular 12 m width (mm) & 8.8 & 0.6 & 8.3 & 0.6 & 0.014 \\
\hline Tibia $6 \mathrm{~m}$ width $(\mathrm{mm})$ & 8.9 & 0.6 & 8.3 & 0.8 & 0.008 \\
\hline Tibia 12 m width (mm) & 9.0 & 0.7 & 8.5 & 0.9 & 0.038 \\
\hline
\end{tabular}

\section{Radiological results}

At 6 months, the mean SNQ index was significantly higher in the Auto group $(12.9 \pm 8.6)$ than in the Allo group $(7.9 \pm 6.3)(p=0.038)$, whereas at 12 months the values were comparable $(9.8 \pm 7.1$ and $10.4 \pm 8.0$ for the Auto and Allo group, respectively) (Fig. 5).

The two-way ANOVA analysis showed that interaction of the two variables was significant $(p=0.022)$, indicating a different behaviour during time depending on the graft type. Indeed while autograft seems to reach a definitive level at 6 months, allograft SNQ slightly increase at
12 months after surgery, despite this difference was not statistically significant.

All the other MRI parameters analyzed are reported in Table 2.

The presence of edema at 12 months was more frequent in patients treated with allograft (15/22) compared to those treated with autograft (13/25), but this difference was not significant (n.s.).

\section{Correlations between SNQ and PROMs}

SNQ score at 6 months did not correlate with Tegner activity scale, ACL-RSI, Cincinnati, VAS or Lysholm score at any of the analysed time-points. The 12-month SNQ values significantly correlate with 60-month Cincinnati $(p=0.003$, $\mathrm{r}=0.442)$, Lysholm $(p=0.027, \mathrm{r}=-0.334)$ and Tegner activity scale $(p=0.018, \mathrm{r}=-0.357)$ (Fig. 6). No correlation was found between 12-month SNQ values and VAS or ACL-RSI 60-month scores.

The attempt to predict 60 -month outcomes considering age, sex, graft type and 12-month SNQ confirmed the role of this radiological score for Tegner activity scale $(p=0.017)$ and Cincinnati score $(p=0.030)$ but not for Lysholm, where male gender played the most significant role in predicting better results $(p=0.027)$. No association was observed between the variables considered and VAS or ACL-RSI recorded at 60 months.

\section{Association between radiological observations and complication/failures, laxity and graft edema}

At 6 months after surgery, patients who then faced complications or re-ruptures had a median SNQ of 11.1 (interquartile range-IQR: 6.6-16.2) compared to the 7.3 (IQR: 4.7-12.6) of patients who did not experienced these events. Nevertheless, this difference was not statistically significant (n.s.), possibly due to the limited number of patients with complications and re-ruptures $(n=3)$. No association was observed considering 12-month SNQ values.

Reported instability at 60 months was not associated with SNQ at 6 or 12 months.

The presence of graft edema at 12 months was associated with reduced sagittal graft inclination $(p=0.020)$ and femoral tunnel width $(p=0.026)$, but not with SNQ value at 6 months.

Femoral and tibial graft width, Blumensaat angle, coronal and sagittal graft inclination angles at 6 months, demonstrated no significant association with the 12- and 60-month Lysholm, TAS and VAS with respect to the two types of graft. 
Fig. 6 Correlations between SNQ at 12 months and Cincinnati score (A), Lysholm score (B) and Tegner Activity Scale (C) at 60 months. While the SNQ score at 6 months did not correlate with any of the clinical and functional score, the 12-month SNQ values significantly correlated with 60 -month Cincinnati $(p=0.003$, $\mathrm{r}=0.442)$, Lysholm $(p=0.027$, $\mathrm{r}=-0.334)$ and Tegner activity scale $(p=0.018, \mathrm{r}=-0.357)$ a

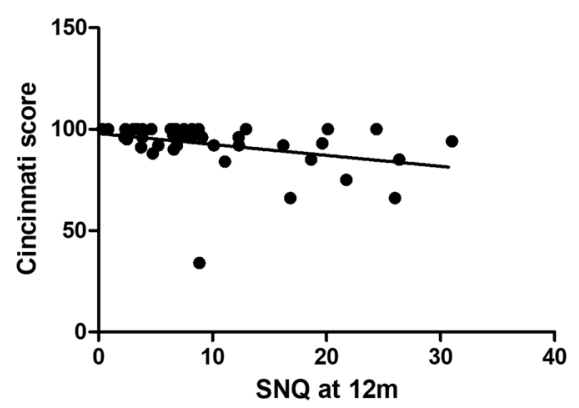

b

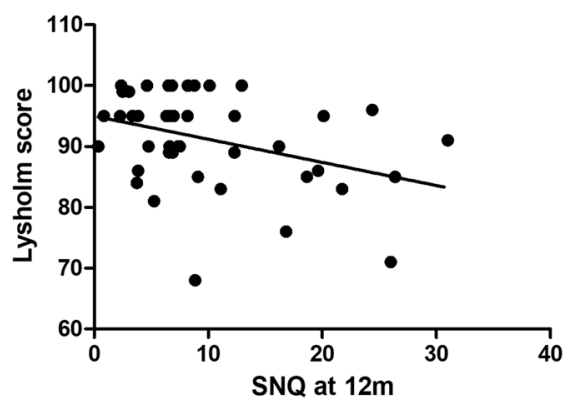

C

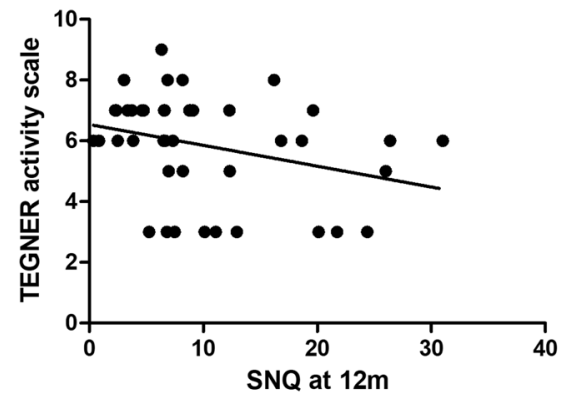

\section{Discussion}

The most important finding of the present study was a different graft maturation time in the two groups, with allografts being more reactive in the first 6 months but with an opposite trend at 12 months, when autografts appeared more mature. Interestingly, the 12-month graft maturation values significantly correlated with Cincinnati score, Lysholm score and Tegner activity scale evaluated at 60-month follow-up. The currently accepted view is that the hamstring autograft detached from its tibial insertion, as used in ACLR, undergoes a process of "ligamentization", showing an initial phase of avascular necrosis with hypocellularity followed by cellular proliferation with high revascularization $[14,25]$. This process, causing changes in graft water content, determines the variation of SNQ values on MRI images. A significant difference in the 6-month SNQ values between the two groups was observed, with autograft patients presenting higher SNQ compared to allograft patients. This difference progressively narrowed during time, with similar SNQ scores between the groups at 12 months. The SNQ mean values for allograft showed a slight increase during time, although not statistically significant, suggesting a slowdown in the maturation process. This finding is in line with previous ones showing higher SNQ for allografts at later follow-ups $[11,19]$. The literature presents controversial data on graft maturation trend. While some studies showed a progressive and persistent increase in the SNQ values of allograft and autograft from 6 to 12 months [24], others reported

an increase in the SNQ for both allograft and autograft at 6 months, followed by a subsequent SNQ decrease [6, 17]. These discrepancies may be related to the differences in the MRI protocols and image evaluation methods as reported by a systematic literature review that concludes that SNQ is still ineffective to anticipate clinical outcomes after ACLR [35].

Several studies examined the association between the graft SNQ and the clinical and functional outcomes [2, 12, $16,17,19,20,26,27]$. Some researchers [12, 17, 26] found no correlation during the first year after surgery comparing SNQ values with objective measurements obtained by KT-1000 arthrometer, while others reported a significant association between graft SNQ and long-term clinical outcomes [16, 27], similarly to what observed in the present study.

Only three studies reported a positive correlation between graft SNQ and clinical results $[2,18,19]$. In particular, Li et al. [19] reported that good graft maturity at 6-month MRI could strongly predict superior clinical results at 1-year follow-up. They also hypothesized the use of SNQ predictive value to improve the functional outcomes and to early detect patients who might need further surgical procedures. However, this study did not confirm this hypothesis, as the SNQ at 6 months did not correlate with any of the clinical scores at any of the time-points.

Interestingly, 12-month SNQ significantly correlated with Cincinnati score, Lysholm score and Tegner activity scale at 60-month follow-up, whereas none of the other MRI parameters did. It is possible that the use of pure axial, sagittal and 
coronal plane to perform such measurements may have negatively influenced these parameters, as it is well known that the graft course is wavy, and measurements may be not fully reliable when performed on standard planes. Further, this study did not highlight any significant association between postoperative complications/failures and 12-month SNQ values.

The secondary aim of this study was to evaluate the suitability of the allograft use in primary ACLR by comparing the clinical-functional recovery of daily and sport activities of patients who underwent this procedure either by autograft or allograft. The evidence provided by old studies showing that allograft ligamentization and integration into host tissue requires a longer time if compared with autograft [26, 34], together with the higher costs of allograft and the poor availability in many countries of allogenic grafts still make autograft the first choice for primary ACLR [23, 34]. Nevertheless, some studies showed no significant clinical and radiological differences between autograft and allograft in primary ACLR $[13,15,22,36-38]$ at the medium-long-term in young active patients.

In this study, all the patients were treated with the same surgical technique and rehabilitation protocol. All the parameters assessed significantly improved over time, with no statistically significant difference between the two groups. The 6-month time point showed lower level of activity in both Auto and Allo group, with a significant improvement at 1-year follow-up and maintained up to 5 years.

ACL-RSI score collected at the last follow-up showed no significant difference between the two groups, indicating a comparable subjective confidence in the operated knee. Similarly, no difference in term of rate and time form surgery of retear events was observed between the two groups.

The satisfaction of the whole study population ( 44 out of 45 patients) meant as the willingness to repeat the same surgery and postoperative rehabilitation protocol to achieve the same functional results, confirmed the effectiveness of the ACLR, regardless of the type of graft.

Study limitations include the subjectivity of all the postoperative clinical and functional evaluations, although the literature confirms that the subjective confidence in the operated knee and the return to daily and sport activities are reliable indicators of good surgical outcomes. A further limitation of the study is the lack of correlation between the radiological parameters and specific sports activities. It would be interesting in the future to restrict the same type of in a more homogeneous population of athletes practicing a specific high-contact sport.

At the time of last follow-up COVID-19 restrictions made not advisable to ask patients to undergo a MRI evaluation. The Authors' intention would be to repeat MRI exams to evaluate further variations in radiological parameters (especially SNQ) several years after surgery.

\section{Conclusion}

The graft maturation time in the two groups was different, with allografts being faster in the first 6 months but appearing less mature than autograft at 12 months.

The significant correlation found between 12-month SNQ with Cincinnati score, Lysholm score and Tegner activity scale collected after 60 months from surgery suggests that MRI can provide an objective evaluation of the graft integration process as well as useful information to predict the best time to return to sport, allowing a more individualized postoperative management.

From a clinical perspective, the findings of this study support the suitability of using allograft in selected cases of primary ACLR as a reasonable alternative to hamstring autografts, given the comparable functional outcomes at short and mid-term follow-up.

Supplementary Information The online version contains supplementary material available at https://doi.org/10.1007/s00167-021-06785-4.

\section{Acknowledgements None.}

Author contribution Conceptualization, AC, PC, and GT; methodology, GR, FF and RP; validation, RP and HS; formal analysis, MV and LdG; investigation, AC, PC, CM and LMS; resources, GT, MV, HS, RP; data curation, FF and GR; patient enrollment, AC, PC, GT, GR, and FF; writing-original draft preparation, $\mathrm{AC}$, $\mathrm{PC}$ and $\mathrm{CM}$; writing-review and editing, $\mathrm{AC}, \mathrm{MV}, \mathrm{LdG}, \mathrm{AZ}$ and GT; visualization, CM, LMS and AZ; supervision, LMS, AZ, RP and HS; project administration, GT and LdG; funding acquisition, HS, RP, LMS and AZ.

Funding This research was funded by Italian Ministry of Health "ricerca corrente".

\section{Declarations}

Conflict of interest Authors declare that there is no conflict of interest.

Ethical approval This study was approved by the Ethics Committee (27/INT/2014) and performed according to the standards EN ISO 14155:1, EN ISO 14155:2, Declaration of Helsinki and Good Clinical Practices (GCP).

Informed consent All study participants, or their legal guardian, provided informed written consent prior to study enrollment.

\section{References}

1. Bait C, Randelli P, Compagnoni R, Ferrua P, Papalia R, Familiari F, Tecame A, Adravanti P, Adriani E, Arnaldi E, Benazzo F, Berruto M, Bonaspetti G, Canata GL, Canè PP, Causero A, Coari G, Denti M, Farè M, Ferretti A, Fravisini M, Giron F, Gobbi A, Madonna V, Manunta A, Mariani PP, Mazzola C, Milano G, Pederzini L, Quaglia F, Ronga M, Schönhuber H, Stefani G, Volpi P, Zanon G, Zini R, Zorzi C, Zaffagnini S 
(2019) Italian consensus statement for the use of allografts in ACL reconstructive surgery. Knee Surg Sports Traumatol Arthrosc 27:1873-1881

2. Biercevicz AM, Akelman MR, Fadale PD, Hulstyn MJ, Shalvoy RM, Badger GJ, Tung GA, Oksendahl HL, Fleming BC (2015) MRI volume and signal intensity of ACL graft predict clinical, functional, and patient-oriented outcome measures after ACL reconstruction. Am J Sports Med 43:693-699

3. Bottoni CR, Smith EL, Shaha J, Shaha SS, Raybin SG, Tokish JM, Rowles DJ (2015) Autograft versus allograft anterior cruciate ligament reconstruction: a prospective, randomized clinical study with a minimum 10-year follow-up. Am J Sports Med 43:2501-2509

4. Briggs KK, Lysholm J, Tegner Y, Rodkey WG, Kocher MS, Steadman JR (2009) The reliability, validity, and responsiveness of the lysholm score and tegner activity scale for anterior cruciate ligament injuries of the knee: 25 years later. Am J Sports Med SAGE Publications Inc STM 37:890-897

5. Cerciello S, Corona K, Morris BJ, Visonà E, Maccauro G, Maffulli N, Ronga M (2018) Cross-cultural adaptation and validation of the Italian versions of the Kujala, Larsen, Lysholm and Fulkerson scores in patients with patellofemoral disorders. J Orthop Traumatol 19(1):18. https://doi.org/10.1186/s10195-018-0508-9

6. Chen L, Wu Y, Lin G, Wei P, Ye Z, Wang Y, Ren T (2018) Graft bending angle affects allograft tendon maturity early after anterior cruciate ligament reconstruction. Knee Surg Sports Traumatol Arthrosc 26:3048-3054

7. Di Leo G, Sardanelli F (2020) Statistical significance: p value, 0.05 threshold, and applications to radiomics-reasons for a conservative approach. Eur Radiol Exp 4:18

8. Ge Y, Li H, Tao H, Hua Y, Chen J, Chen S (2015) Comparison of tendon-bone healing between autografts and allografts after anterior cruciate ligament reconstruction using magnetic resonance imaging. Knee Surg Sports Traumatol Arthrosc 23:954-960

9. Goetz G, de Villiers C, Sadoghi P, Geiger-Gritsch S (2020) Allograft for Anterior Cruciate Ligament Reconstruction (ACLR): a systematic review and meta-analysis of long-term comparative effectiveness and safety. results of a health technology assessment. Arthrosc Sports Med Rehabil 2:e873-e891

10. Grassi A, Bailey JR, Signorelli C, Carbone G, TchonangWakam A, Lucidi GA, Zaffagnini S (2016) Magnetic resonance imaging after anterior cruciate ligament reconstruction: A practical guide. World J Orthop 7:638-649

11. van Groningen B, van der Steen MC, Janssen DM, van Rhijn LW, van der Linden AN, Janssen RPA (2020) Assessment of graft maturity after anterior cruciate ligament reconstruction using autografts: a systematic review of biopsy and magnetic resonance imaging studies. Arthrosc Sports Med Rehabil 2:e377-e388

12. Hofbauer M, Soldati F, Szomolanyi P, Trattnig S, Bartolucci F, Fu F, Denti M (2019) Hamstring tendon autografts do not show complete graft maturity 6 months postoperatively after anterior cruciate ligament reconstruction. Knee Surg Sports Traumatol Arthrosc 27:130-136

13. Hulet C, Sonnery-Cottet B, Stevenson C, Samuelsson K, Laver L, Zdanowicz U, Stufkens S, Curado J, Verdonk P, Spalding T (2019) The use of allograft tendons in primary ACL reconstruction. Knee Surg Sports Traumatol Arthrosc 27:1754-1770

14. Janssen RPA, Scheffler SU (2014) Intra-articular remodelling of hamstring tendon grafts after anterior cruciate ligament reconstruction. Knee Surg Sports Traumatol Arthrosc 22:2102-2108

15. Kan S-L, Yuan Z-F, Ning G-Z, Yang B, Li H-L, Sun J-C, Feng S-Q (2016) Autograft versus allograft in anterior cruciate ligament reconstruction: A meta-analysis with trial sequential analysis. Medicine (Baltimore) 95:e4936

16. Lee S, Seong SC, Jo CH, Han HS, An JH, Lee MC (2007) Anterior cruciate ligament reconstruction with use of autologous quadriceps tendon graft. J Bone Joint Surg Am 89(Suppl 3):116-126
17. Li H, Chen J, Li H, Wu Z, Chen S (2017) MRI-based ACL graft maturity does not predict clinical and functional outcomes during the first year after ACL reconstruction. Knee Surg Sports Traumatol Arthrosc 25:3171-3178

18. Li H, Chen S, Tao H, Li H, Chen S (2014) Correlation analysis of potential factors influencing graft maturity after anterior cruciate ligament reconstruction. Orthop J Sports Med 2(10):2325967114553552. https://doi.org/10.1177/2325967114 553552

19. Li Q, Zhang Y, Zhan L, Han Q, Wu M, Zhang N (2019) Correlation analysis of magnetic resonance imaging-based graft maturity and outcomes after anterior cruciate ligament reconstruction using international knee documentation committee score. Am J Phys Med Rehabil 98:387-391

20. Liu S, Li H, Tao H, Sun Y, Chen S, Chen J (2018) A randomized clinical trial to evaluate attached hamstring anterior cruciate ligament graft maturity with magnetic resonance imaging. Am J Sports Med 46:1143-1149

21. Mahapatra P, Horriat S, Anand BS (2018) Anterior cruciate ligament repair - past, present and future. J Exp Ortop 5:20. https:// doi.org/10.1186/s40634-018-0136-6

22. Mariscalco MW, Magnussen RA, Mehta D, Hewett TE, Flanigan DC, Kaeding CC (2014) Autograft versus nonirradiated allograft tissue for anterior cruciate ligament reconstruction: a systematic review. Am J Sports Med 42:492-499

23. Mistry H, Metcalfe A, Colquitt J, Loveman E, Smith NA, Royle P, Waugh N (2019) Autograft or allograft for reconstruction of anterior cruciate ligament: a health economics perspective. Knee Surg Sports Traumatol Arthrosc 27:1782-1790

24. Muramatsu K, Hachiya Y, Izawa H (2008) Serial evaluation of human anterior cruciate ligament grafts by contrast-enhanced magnetic resonance imaging: comparison of allografts and autografts. Arthroscopy 24:1038-1044

25. Pauzenberger L, Syré S, Schurz M (2013) "Ligamentization" in hamstring tendon grafts after anterior cruciate ligament reconstruction: a systematic review of the literature and a glimpse into the future. Arthroscopy 29:1712-1721

26. Rose M, Crawford D (2017) Allograft maturation after reconstruction of the anterior cruciate ligament is dependent on graft parameters in the sagittal plane. Orthop J Sports Med 5:2325967117719695

27. Saupe N, White LM, Chiavaras MM, Essue J, Weller I, Kunz M, Hurtig M, Marks P (2008) Anterior cruciate ligament reconstruction grafts: MR imaging features at long-term follow-upcorrelation with functional and clinical evaluation. Radiology 249:581-590

28. Shelbourne KD, Barnes AF, Gray T (2012) Correlation of a single assessment numeric evaluation (SANE) rating with modified Cincinnati knee rating system and IKDC subjective total scores for patients after ACL reconstruction or knee arthroscopy. Am J Sports Med 40:2487-2491

29. Spalding T, Verdonk P, de Girolamo L, Seil R, Dejour D (2019) Allografts in joint reconstruction: ESSKA making a difference. Knee Surg Sports Traumatol Arthrosc 27:1701-1703

30. Strauss MJ, Varatojo R, Boutefnouchet T, Condello V, Samuelsson K, Gelber PE, Adravanti P, Laver L, Dimmen S, Eriksson K, Verdonk P, Spalding T (2019) The use of allograft tissue in posterior cruciate, collateral and multi-ligament knee reconstruction. Knee Surg Sports Traumatol Arthrosc 27:1791-1809

31. Takahashi T, Kimura M, Takeshita K (2017) MRI evaluation of the ACL remnant tissue in ACL-deficient knee. J Orthop Surg (Hong Kong) 25:2309499017739479

32. Thiebat G, Cucchi D, Spreafico A, Muzzi S, Viganò M, Visconti L, Facchini F, de Girolamo L (2021) Italian version of the anterior cruciate ligament-return to sport after injury scale (IT ACL-RSI): translation, cross-cultural adaptation, validation and ability to 
predict the return to sport at medium-term follow-up in a population of sport patients. Knee Surg Sports Traumatol Arthrosc. https://doi.org/10.1007/s00167-021-06498-8

33. Thong ISK, Jensen MP, Miró J, Tan G (2018) The validity of pain intensity measures: what do the NRS, VAS, VRS, and FPS-R measure? Scand J Pain 18:99-107

34. Tisherman R, Wilson K, Horvath A, Byrne K, De Groot J, Musahl V (2019) Allograft for knee ligament surgery: an American perspective. Knee Surg Sports Traumatol Arthrosc 27:1882-1890

35. Van Dyck P, Zazulia K, Smekens C, Heusdens CHW, Janssens T, Sijbers J (2019) Assessment of Anterior Cruciate Ligament Graft Maturity With Conventional Magnetic Resonance Imaging: A Systematic Literature Review. Orthop J Sports Med 7:2325967119849012

36. Wang H-D, Zhu Y-B, Wang T-R, Zhang W-F, Zhang Y-Z (2018) Irradiated allograft versus autograft for anterior cruciate ligament reconstruction: A meta-analysis and systematic review of prospective studies. Int J Surg 49:45-55

37. Wang S, Zhang C, Cai Y, Lin X (2018) Autograft or allograft? irradiated or not? a contrast between autograft and allograft in anterior cruciate ligament reconstruction: a meta-analysis. Arthroscopy 34:3258-3265

38. Zeng C, Gao S, Li H, Yang T, Luo W, Li Y, Lei G (2016) Autograft versus allograft in anterior cruciate ligament reconstruction: a meta-analysis of randomized controlled trials and systematic review of overlapping systematic reviews. Arthroscopy 32:153163.e18. https://doi.org/10.1016/j.arthro.2015.07.027

Publisher's Note Springer Nature remains neutral with regard to jurisdictional claims in published maps and institutional affiliations.

\section{Authors and Affiliations}

\section{Andrea Cusumano ${ }^{1}$. Paolo Capitani ${ }^{2}$. Carmelo Messina ${ }^{1}$. Laura de Girolamo ${ }^{1} \cdot$ Marco Viganò $^{1}$. Giovanni Ravasio ${ }^{1}$. Francesca Facchini ${ }^{1}$. Luca Maria Sconfienza ${ }^{1,3}$ - Alberto Zerbi ${ }^{4} \cdot$ Herbert Schoenhuber $^{1} \cdot$ Roberto Pozzoni $^{1}$. Gabriele Thiébat ${ }^{1}$ (D)}

1 IRCCS Istituto Ortopedico Galeazzi, Via R. Galeazzi 4, 20161 Milan, Italy

2 Department of Orthopedic Surgery and Traumatology, ASST GOM Niguarda, Piazza dell'Ospedale Maggiore, 3, 20162 Milan, Italy
3 Present Address: Dipartimento di Scienze Biomediche per la Salute, Università degli Studi di Milano, Via Festa del Perdono, 7, 20122 Milan, Italy

4 Casa Di Cura La Madonnina, Via Quadronno, 29, 20122, Milan, Italy 